\title{
Cómo han vivido la pandemia las personas con discapacidad visual durante la primera y segunda ola: sus preocupaciones y su estado anímico
}

\author{
The first and second waves of the pandemic \\ as experienced by people with visual disabilities: \\ concerns and state of mind
}

\section{V.O. Dabbagh Rollán}

\section{Resumen}

La crisis provocada por la covid-19 ha afectado a todas las personas, pero no a todas por igual. Así mismo, muchos son los ámbitos sobre los que se pueden ver los efectos que ha provocado esta pandemia. En este artículo se presenta parte de los resultados de un estudio más amplio que se llevó a cabo a finales de 2020 por parte de la ONCE y que tenía por objetivo analizar los efectos de la covid-19 entre las personas con discapacidad visual afiliadas a la ONCE. En concreto, se presentan las principales conclusiones extraídas del análisis realizado sobre cómo la pandemia, durante la primera y segunda ola, ha afectado al estado anímico de las personas con ceguera o deficiencia visual, así como las principales preocupaciones de estas durante este periodo; preocupaciones y estados anímicos que, aunque son coincidentes con los de la población en general, adquieren matices importantes a tener en cuenta, matices en cuanto a frecuencia, intensidad e implicaciones derivadas de los mismos.

\section{Palabras clave}

Discapacidad visual. Covid-19. Preocupaciones sociales. Estado anímico. 


\begin{abstract}
The crisis triggered by covid-19 has affected everyone, but not everyone to the same degree. The impact of the pandemic is visible in any number of realms. This article describes the findings of a broad survey conducted by ONCE in late 2020 to analyse the effects of covid-19 on affiliates with visual disability. More specifically, it discusses the key conclusions drawn from the analysis of how the first and second waves of the pandemic have affected the state of mind of blind or visually impaired people, along with their main concerns while it raged. Although such concerns and mental attitudes were similar to those of the population at large, the survey revealed certain significant nuances in terms of frequency, intensity and implications that must be borne in mind.
\end{abstract}

\title{
Key words
}

Visual impairment. Covid-19. Social concerns. State of mind.

\section{Introducción}

El 2020 pasará a la historia, sin lugar a dudas, por la crisis provocada por la covid-19, que ha traído consigo una paralización de la actividad económica, un cambio en los hábitos cotidianos de las personas, en su forma de desplazarse y relacionarse... todos ellos elementos generadores de estrés y que han convertido a la incertidumbre en un elemento habitual en el día a día de cualquier persona dentro de la llamada «nueva normalidad».

Uno de los ámbitos donde más se han hecho notar los efectos de la pandemia ha sido en el emocional. Como señalan distintos estudios (Marroquín et al., 2020; Ammar et al., 2021; Cénat et al., 2021), la covid-19 ha tenido una incidencia notable en la salud mental y en el estado anímico de las personas. Para el caso español, son referentes las encuestas llevadas a cabo por el Centro de Investigaciones Sociológicas (CIS) durante el 2020 y 2021, y que ponen de manifiesto cómo la mayoría de las y los españoles se muestran preocupados por la situación actual provocada por la covid-19 y cómo gran parte muestran estados de ánimo disfóricos, como depresión, tristeza, depresión, ansiedad... (CIS, 2020a, 2020b, 2021).

Pero hay que tener en cuenta que si bien la nueva situación generada por el coronavirus repercute en la salud tanto física como psíquica de todas las personas, esta

Dabbagh, V.O. (2021). Cómo han vivido la pandemia las personas con discapacidad visual durante la primera y segunda ola: sus preocupaciones y su estado anímico. RED Visual: Revista Especializada en Discapacidad Visual, 77, 13-42. https://doi.org/10.53094/XBUN9999. 
incide especialmente en los colectivos y personas más vulnerables, es decir, «aquéllas [sic] que por sus características presentan una desventaja por edad, sexo, estructura familiar, nivel educativo, origen étnico, situación o condición física y/o mental, y que requieran de un esfuerzo adicional para incorporarse al desarrollo y a la convivencia» (Balluerka et al., 2020, p. 7). En este sentido, la Organización Mundial de la Salud (OMS) advierte de que el colectivo de personas con discapacidad es esencialmente vulnerable en tanto que deben hacer frente a una serie de obstáculos para mantener el distanciamiento social, acceder a la información disponible y a la atención de salud, para seguir las pautas de higiene, etc. (OMS, 2020), y se advierte (European Union Agency for Fundamental Rights, 2020) de que este colectivo corre el riesgo de ver cómo servicios y apoyos de vital importancia para ellos han sido suspendidos o interrumpidos.

Precisamente distintos estudios, entre ellos los de Brennan (2020) y Silván y Quífez (2020), han evidenciado cómo la covid-19 ha repercutido especialmente en las personas con discapacidad, dificultando su acceso a distintos servicios (como la atención médica), impidiendo el ejercicio de sus libertades y derechos, aislándolas respecto al resto de la sociedad, imposibilitando su formación, el ejercicio profesional o el acceso al empleo, etc., todo lo cual redunda en un malestar no solo físico, sino también emocional, en tanto que las distintas barreras a las que debe enfrentarse una persona con discapacidad son potenciales generadores de estrés que se añaden a las que comparten con la población en general y que repercuten directamente en su estado anímico.

Además, se ha de tener en cuenta que el impacto de la pandemia no ha tenido la misma repercusión en todas las personas con discapacidad y no se han tenido en cuenta las especificidades de determinados grupos poblacionales (Brennan, 2020). Por ello, se hace necesaria la realización de estudios que centren su atención en colectivos concretos que permitan identificar las particularidades de los mismos de cara a poder desarrollar actuaciones específicas que redunden en el bienestar de la gente de manera más efectiva.

Para el colectivo de personas con ceguera o deficiencia visual, diferentes entidades de y para personas con discapacidad visual han desarrollado distintos estudios durante el 2020 -como los realizados por la World Blind Union (Zayed et al., 2020), el Canadian Council of the Blind (Gordon, 2020), el Royal National Institute of Blind People (Bertiz y Dearing, 2020) o la American Foundation for the Blind (Rosenblum et

Dabbagh, V.O. (2021). Cómo han vivido la pandemia las personas con discapacidad visual durante la primera y segunda ola: sus preocupaciones y su estado anímico. RED Visual: Revista Especializada en Discapacidad Visual, 77, 13-42. https://doi.org/10.53094/XBUN9999. 
al., 2020) - para analizar cuáles han sido las barreras y retos a los que se han tenido que enfrentar las personas con discapacidad visual durante la pandemia, así como los efectos que ha tenido sobre ellas. El presente artículo se centra en cómo la covid-19 ha afectado al estado de ánimo de las personas con discapacidad visual en España y en analizar cuáles son sus preocupaciones respecto a la misma.

\section{Metodología}

A finales de 2020, la ONCE, bajo la coordinación de la Asesoría de Servicios Sociales, llevó a cabo un estudio para conocer cómo había afectado y estaba afectando la crisis provocada por el coronavirus a sus afiliados y afiliadas. Para la realización de dicho estudio, del que se han extraído las conclusiones para la redacción de este artículo, se diseñó una metodología mixta que incluía tanto la utilización de técnicas cuantitativas como cualitativas.

En primer lugar, de cara a obtener información representativa del colectivo de personas con discapacidad visual mayores de edad, se optó por realizar una encuesta telefónica entre una muestra representativa de las personas afiliadas a la ONCE a mediados del mes de octubre. Para dicha encuesta se establecieron cuotas de edad, sexo y condición visual. Así mismo, se fijó una cuota mínima de encuestas en función del tamaño de hábitat. Conjuntamente a dicha encuesta, y en la línea de esta, se realizaron una serie de entrevistas estructuradas adaptadas a personas afiliadas con sordoceguera de tal manera que se logró obtener una muestra total de 1107 personas, cuya composición se puede ver en la Tabla 1.

Tabla 1. Reparto de la muestra en función de distintos perfiles

\begin{tabular}{|l|r|}
\hline \multicolumn{1}{|c|}{ Perfil } & \multicolumn{2}{|c|}{$\begin{array}{c}\text { Porcentaje dentro } \\
\text { de la muestra }\end{array}$} \\
\hline Hombre & 47,8 \\
\hline Mujer & 52,2 \\
\hline 18-34 años & 7,8 \\
\hline 35-64 años & 42,7 \\
\hline 65-74 años & 18,5 \\
\hline 75 o más años & 31,0 \\
\hline
\end{tabular}

Dabbagh, V.O. (2021). Cómo han vivido la pandemia las personas con discapacidad visual durante la primera y segunda ola: sus preocupaciones y su estado anímico. RED Visual: Revista Especializada en Discapacidad Visual, 77, 13-42. https://doi.org/10.53094/XBUN9999. 


\begin{tabular}{|l|r|}
\hline \multicolumn{1}{|c|}{ Perfil } & \multicolumn{2}{|c|}{$\begin{array}{c}\text { Porcentaje dentro } \\
\text { de la muestra }\end{array}$} \\
\hline Ceguera total & 16,1 \\
\hline Deficiencia visual grave & 83,9 \\
\hline Con sordoceguera & 4,0 \\
\hline Sin sordoceguera & 96,0 \\
\hline
\end{tabular}

$n: 1107$.

Paralelamente a la encuesta, se consideró conveniente la inclusión en el estudio de entrevistas semiestructuradas de cara tanto a profundizar en los resultados obtenidos como para contar con el discurso de otros agentes que no se habían incluido en la metodología cuantitativa.

La selección de las personas a entrevistar obedeció a la identificación de perfiles clave durante el proceso iniciado con el confinamiento del pasado mes de marzo y buscó la representación territorial. En total, se realizaron 24 entrevistas.

El primer nivel de definición de los perfiles a entrevistar hacía referencia a la condición de afiliado y edad, ser familiar de afiliado o profesional de la ONCE (ver Tabla 2). En un segundo nivel se introdujeron otra serie de variables, como ocupación, tamaño de hábitat o discapacidades asociadas.

Tabla 2. Reparto de las entrevistas en función del primer nivel de definición de los perfiles

\begin{tabular}{|l|l|}
\hline \multicolumn{1}{|c|}{ Perfiles generales } & Número de entrevistas \\
\hline Afiliados entre 18 y 35 años & 4 entrevistas \\
\hline Afiliados entre 36 y 54 años & 4 entrevistas \\
\hline Afiliados de 55 y más años & 4 entrevistas \\
\hline Familiares de afiliados & 4 entrevistas \\
\hline $\begin{array}{l}\text { Profesionales/voluntarios de la oncE que participan } \\
\text { o colaboran en programas activos durante } \\
\text { el confinamiento }\end{array}$ & 8 entrevistas \\
\hline
\end{tabular}

Dabbagh, V.O. (2021). Cómo han vivido la pandemia las personas con discapacidad visual durante la primera y segunda ola: sus preocupaciones y su estado anímico. RED Visual: Revista Especializada en Discapacidad Visual, 77, 13-42. https://doi.org/10.53094/XBUN9999. 
El análisis de la información recabada a partir de las entrevistas se realizó atendiendo a grupos temáticos de los discursos de los participantes, agrupando, codificando y categorizando la información obtenida en función de los distintos temas de análisis y del perfil de la persona informante.

\section{Principales resultados}

De cara a hacer una mejor presentación de los resultados obtenidos, en primer lugar, se presentarán los referidos a las principales preocupaciones de las personas con discapacidad visual con respecto a la covid-19, para, posteriormente, centrar la atención en el estado anímico de las mismas durante la pandemia.

\subsection{Principales preocupaciones de las personas con discapacidad visual}

La primera cuestión por la que se quiso indagar era el grado de preocupación existente respecto al propio coronavirus. En este sentido, se puede apreciar (Tabla 3) cómo la inmensa mayoría de las personas encuestadas, un $87 \%$, manifiesta sentir mucha o bastante preocupación.

Tabla 3. Grado de preocupación de las personas con discapacidad visual respecto al coronavirus (\%)

\begin{tabular}{|l|r|}
\hline Grado de preocupación & \multicolumn{2}{|c|}{ Porcentaje } \\
\hline Mucho & 47,3 \\
\hline Bastante & 39,7 \\
\hline Poco & 7,8 \\
\hline Nada & 3,3 \\
\hline NS/NC & 1,9 \\
\hline
\end{tabular}

n: 1107.

Analizando estos resultados en función de la edad, se obtiene que quienes tienen una mayor preocupación respecto a la covid-19 es el grupo de personas de entre 55 y 64 años, mientras que quienes muestran una menor preocupación son el colectivo de personas de entre 18 y 34 años; resultados, por otro lado, semejantes a los obtenidos 
para el conjunto de la población española tal y como se desprende del barómetro del CIS de octubre de $2020 .^{1}$

La covid-19, como se ha comentado anteriormente, ha afectado a las personas de distintas maneras y en distintos ámbitos de la vida. Por ello, se quiso desgranar esta preocupación manifestada por el coronavirus, empezando por la salud. En este sentido, el $64 \%$ las personas encuestadas han señalado que están preocupadas ante la posibilidad de enfermar (Tabla 4).

En los discursos de las y los entrevistados se ha puesto en evidencia cómo dicha preocupación es más intensa entre quienes parten de un estado de salud afectado por enfermedades previas y quiénes podrían tener mayores dificultades para recibir la atención sanitaria necesaria.

Cuadro 1. Comentarios extraídos de las entrevistas realizadas sobre la preocupación a enfermar

«Una cierta preocupación por mí, porque yo tengo un diagnóstico. Estoy muy bien de salud, pero tengo un diagnóstico neurológico que, a día de hoy, no sé si se puede considerar de riesgo o no. Y también me rondaba como una cierta preocupación de si solo faltaría que lo cogiera, que esto se agravara o que tal. Eso, bueno, no le doy muchas vueltas, pero siempre ha estado ahí como un poco de una cierta inquietud».

\section{Persona afiliada de entre 35-54 años.}

«Porque a veces tengo síntomas y no sé si son debidos al coronavirus o a otra cosa, y yo, con mi discapacidad auditiva, me preocupa ponerme malo y no poder tener acceso al médico».

\section{Persona afiliada de más de 54 años con sordoceguera.}

«Muchos de ellos con mucho aislamiento y miedo a qué iba a pasar después de todo esto, y si le iba a afectar a nivel de sus enfermedades, porque claro, nosotros nos movemos con personas que, aparte del tema de la sordoceguera, tienen múltiples problemas, bien porque son muy mayores y ya se acumulan las patologías, bien porque son personas afectadas de algún síndrome que, bueno, el tema visual y auditivo no es que sea secundario, pero una patología más dentro de sus múltiples discapacidades, dentro de sus múltiples enfermedades y síntomas, claro».

Profesional de la ONCE - Ámbito sordoceguera.

1 El CIS ha llevado distintos barómetros a lo largo de 2020 en los que ha incluido la cuestión del coronavirus entre sus preguntas. Se ha seleccionado el del mes de octubre para la comparación con los datos obtenidos en este estudio porque el trabajo de campo en ambos se realizó en el mismo mes.

Dabbagh, V.O. (2021). Cómo han vivido la pandemia las personas con discapacidad visual durante la primera y segunda ola: sus preocupaciones y su estado anímico. RED Visual: Revista Especializada en Discapacidad Visual, 77, 13-42. https://doi.org/10.53094/XBUN9999. 
Pero la preocupación aumenta cuando en quien se piensa es en el entorno cercano. De esta manera, el $88,3 \%$ de las personas entrevistadas (Tabla 4) señalan que están preocupadas ante la posibilidad de que algún pariente o conocido enferme. $^{2}$

Tabla 4. Preocupaciones de las personas con discapacidad visual en relación al coronavirus - salud (\%)

\begin{tabular}{|l|c|}
\hline \multicolumn{1}{|c|}{ Preocupaciones } & \multicolumn{2}{c|}{ Porcentaje } \\
\hline Preocupación a enfermar & 64,0 \\
\hline $\begin{array}{l}\text { Preocupación a que enferme } \\
\text { algún familiar o conocido }\end{array}$ & 88,3 \\
\hline
\end{tabular}

$n: 1107$.

Cuadro 2. Comentarios extraídos de las entrevistas realizadas sobre la preocupación a que enfermen las personas allegadas

«En cuanto a la salud, estaba un poco preocupado por todos».

\section{Persona afiliada de entre 18-34 años.}

«Tengo mucho miedo a que la gente enferme y también mi familia».

\section{Persona afiliada de entre 35-54 años.}

«Lo primero que temían era a su seguridad y a la de los suyos».

\section{Profesional de la ONCE - Tiflotecnología.}

Este miedo o preocupación a enfermar lleva aparejado distintos comportamientos, como, por ejemplo, posponer los servicios sanitarios que la propia persona no considera como esenciales o vitales, con la consecuente repercusión que pueda tener en la salud personal. La persona hace una valoración en la que contrapone el riesgo a contraer la covid-19 con la repercusión que puedan tener sobre su salud otras enfermedades que se están manifestando, valoración en la que en no en pocas ocasiones pesa más el miedo al coronavirus.

\footnotetext{
2 Porcentaje similar (89,9\%) ha obtenido Gordon (2020) para la población canadiense en su estudio realizado en abril
} de 2020.

Dabbagh, V.O. (2021). Cómo han vivido la pandemia las personas con discapacidad visual durante la primera y segunda ola: sus preocupaciones y su estado anímico. RED Visual: Revista Especializada en Discapacidad Visual, 77, 13-42. https://doi.org/10.53094/XBUN9999. 
Cuadro 3. Comentarios extraídos de las entrevistas realizadas sobre el aplazamiento voluntario de la atención sanitaria

«En marzo tenía una analítica anual, pues de revisión anual, y lo que he hecho ha sido posponerla pues hasta hace 15 días, porque, bueno, no era necesario».

\section{Persona afiliada de 55 o más años.}

«Mira, si yo supiera que ir a la consulta de Madrid fuera imprescindible y la iba a mejorar en algo... Porque además eso, tiene la de neuropediatría en el HUCA de Oviedo y tiene un montón de consultas. Entonces, si yo supiera que todo eso iba a servir para algo yo iría ahora a Madrid, ahora en diciembre, que nos toca, y si le van a hacer algo que la va a mejorar, por supuesto que iría. Por supuesto que sí.

»Pero que están haciéndole estudios y los estudios van a ser lo mismo este año que el año que viene. Exactamente igual».

\section{Familiar persona afiliada menor de edad.}

«Sobre todo a nivel físico, más que a nivel emocional a nivel físico, a nivel de estado de salud, a nivel de incertidumbre, de acceder o no a los servicios de salud, al miedo de "a mí me duele, pero me voy a esperar un poquito porque es que yo no quiero ir, a ver si me voy a coger lo que no tengo"».

Profesional de la onCE - Trabajo social.

Cuadro 4. Comentarios extraídos de las entrevistas realizadas sobre el aplazamiento forzoso de la atención sanitaria

«Lo de la salud me preocupa, porque no puedes conectar con los médicos. Yo no he podido contactar con mi doctora, no he podido ir en dos semanas, y tampoco he podido contactar por teléfono ni que me trate [...]. He tenido miedo a morir de cáncer sin que me atendieran».

\section{Persona afiliada de más de 54 años.}

«Preocupación porque el acceso a sanidad ha quedado muy restringido y es muy difícil conseguir citas para cualquier problema de salud que me pueda surgir».

\section{Persona afiliada de más de 54 años con sordoceguera.}

«Sus citas con los médicos se han suspendido o se han retrasado, porque, como te digo, no solamente son invidentes, tienen otras enfermedades $u$ otras discapacidades [...]. Estaba pendiente de una biopsia, y se la habían retrasado por el tema del coronavirus y estaba muy preocupada».

\section{Persona voluntaria en la ONCE.}

Dabbagh, V.O. (2021). Cómo han vivido la pandemia las personas con discapacidad visual durante la primera y segunda ola: sus preocupaciones y su estado anímico. RED Visual: Revista Especializada en Discapacidad Visual, 77, 13-42. https://doi.org/10.53094/XBUN9999. 
Es importante no confundir el aplazamiento voluntario de ciertas atenciones sanitarias, que es a lo que se ha hecho referencia en el punto anterior, al aplazamiento forzoso provocado por la situación sanitaria en los centros de salud. Dicho aplazamiento no se vive como un alivio, sino, al contrario, como un factor generador de estrés que agrava las preocupaciones que las personas puedan tener sobre otras cuestiones relativas a la salud más allá de la covid. Esto es de importancia teniendo en cuenta que el $24,1 \%$ de las personas encuestadas han manifestado que no han recibido la atención sanitaria suficiente y el $16 \%$ solo a veces.

Cuadro 5. Comentarios extraídos de las entrevistas realizadas sobre el aplazamiento voluntario de los servicios de la ONCE

«Al señor mayor al que le llamo y le digo: "Mire, que me han dicho que está usted interesado en manejar un móvil y tendríamos que vernos..."; "Ay, hija, es que yo prefiero cuando pase esto un poco". Esto nos lo han dicho, no te digo todos, pero sí que hay gente [...]. Luego hay otras personas que no, que no tienen esa cuestión personal».

\section{Profesional de la ONCE - Tiflotecnología.}

«A mí prácticamente nadie me quiere venir a una cita presencial [...], y a mí me posponen un servicio para cuando se normalice la situación, para cuando haya una vacuna».

\section{Profesional de la onCE - Trabajo social.}

«Mi apreciación es que se solicitan menos, no se ponen tanto en contacto con la ONCE para solicitar tantas cosas, no sé si por el temor de salir a la calle o por el tema del contagio, porque son personas que tienen un mayor riesgo de contagio y entonces a lo mejor se reservan más».

\section{Persona voluntaria en la ONCE.}

«Nos encontramos la situación de que buena parte de los afiliados a los que se les cita ponen pegas para desplazarse, bien a la agencia o bien para nosotros ser recibidos en su propio domicilio, porque dicen: "No, no, aquí en mi casa nadie entra". Perfecto, respeto al máximo, obviamente».

\section{Profesional de la ONCE - Ámbito sordoceguera.}

Junto a los servicios sanitarios, también hay otra serie de servicios que redundan en la calidad de vida de las personas con discapacidad visual y que se han visto aplazados por ese miedo al posible contagio, servicios como son los prestados por 
los distintos profesionales de los servicios sociales de la ONCE. En este caso, el perfil de las personas que realizan este tipo de aplazamiento voluntario se corresponde, mayoritariamente, con el de las personas mayores o de personas afiliadas a la ONCE recientemente, que todavía no han recibido con anterioridad el servicio que demandan o que necesitan.

Otra de las preocupaciones por las que se quiso indagar fue el miedo a salir de casa. Tal como se observa en la Tabla 5, la mayoría de las personas encuestadas, el 51\%, han manifestado estar preocupados al respecto, siendo el colectivo de 18 a 34 años el que menos preocupación ha mostrado al respecto y las personas con sordoceguera las que más.

Tabla 5. Preocupaciones de las personas con discapacidad visual en relación al coronavirus salir a la calle (\%)

\begin{tabular}{|l|r|}
\hline \multicolumn{1}{|c|}{ Perfil } & Porcentaje \\
\hline Población general & 50,9 \\
\hline Población entre 18-34 años & 37,2 \\
\hline Población con miedo a contagiarse & 60,0 \\
\hline Población sin miedo a contagiarse & 24,9 \\
\hline
\end{tabular}

n: 1107.

Es importante señalar cómo la preocupación por salir a la calle tiene dos vertientes. En primer lugar, tal como también señala el estudio realizado por el Canadian Council of the Blind (Gordon, 2020), la relacionada con la posibilidad de contagiarse por el mero hecho de abandonar la seguridad del hogar y entrar en contacto con otras personas. En este sentido, atendiendo solo a las personas que tienen miedo a contagiarse, el porcentaje de quienes manifiestan tener miedo a salir a la calle aumenta hasta el $60 \%$.

Es necesario puntualizar que esta preocupación, común en el conjunto de la población, se agrava en las personas con discapacidad visual, paras las que, tal y como señala la OMS (2020), el tacto es un elemento necesario de cara a obtener información del entorno y poder relacionarse con él. 
Cuadro 6. Comentarios extraídos de las entrevistas realizadas sobre el miedo a salir a la calle (1)

«Entonces voy con él, pero siempre vas con ese miedo, siempre vas por la calle que no me quiero rozar con nadie, siempre estás dentro de un supermercado o, a ver, esta persona está en la estantería, yo necesito coger esto... Hay personas que vamos con más prisa y otras personas que van más relajadas, Yo estoy deseando entrar, coger y salir. Me agobia, es como una sensación de agobio el entrar en cualquier sitio».

\section{Persona afiliada de 55 o más años, jubilada/pensionista.}

«Sí, ellos tenían el miedo de ir al cole también. Claro, es que aquí estábamos como muy encerrados, muy protegidos».

\section{Familia persona afiliada menor de edad.}

«Y luego también el miedo que genera en algunos niños [...]. Niños que no querían salir».

\section{Profesional de la ONCE - Ámbito educativo.}

«Nos vamos a la desescalada. Ya desde el equipo técnico, los profesionales, ya preveíamos, ya lo veíamos, los miedos a salir a la calle, a tocar, a ser tocado, a ese contagio, ese posible rechazo a ir tocando por la calle y me van a decir o me van a dejar de decir..., que sí que se ha dado [...]. Y sí que me encontré con algunos casos de gente que le costaba salir precisamente porque había riesgos..., sobre todo en casos de ciegos totales y en zonas donde hay que utilizar el transporte público. Unos temores que ahí sí había una diferencia fundamental con el resto de la población. El miedo a tocar, a ser tocado, no controlar».

\section{Profesional de la ONCE - Apoyo psicológico.}

Además, la intranquilidad que genera el salir a la calle, por su asociación con el riesgo a enfermar, se agrava ante el comportamiento observado en parte de la población al no cumplir con las medidas establecidas. Incluso aunque no se observen estos comportamientos, el hecho de estar escuchando incesantemente en las noticas y medios de comunicación cómo los mismos se dan, hace que el temor aumente, más cuando muchas personas con discapacidad visual no tienen resto visual suficiente como para ver a relativa distancia si las personas con las que pueden compartir espacios o con las que se pueden cruzar en la calle están cumpliendo o no con la obligación, por ejemplo, de llevar la mascarilla puesta o de mantener la «distancia social».

Como se comentara anteriormente, la preocupación por salir a la calle no solamente está relacionada con la posibilidad de contagiarse. Otro de los aspectos a los que se hace referencia es a las nuevas barreras que han surgido en el entorno físico que 
dificultan el desplazamiento de las personas con discapacidad visual al introducirse modificaciones en los recorridos habituales, que, como en cualquier situación en la que se introducen elementos inusuales, implican una pérdida de habilidades en cuanto a desplazamiento se refiere (Jacobson, 1993) o la necesidad de iniciar un proceso de reorientación (Ortiz, 2011). Se ha de recordar, a modo de ejemplo de este tipo de alteraciones espaciales, cómo se permitió a bares y restaurantes ampliar sus terrazas cuando el acceso al interior de estos establecimientos estaba prohibido, lo que provocó una reducción del espacio peatonal.

Cuadro 7. Comentarios extraídos de las entrevistas realizadas sobre el miedo a salir a la calle (2)

«Porque entre el no ver y pensar en el que lleva o no lleva mascarilla, eso también te crea unas dudas...».

\section{Persona afiliada de entre 35 y 54 años.}

«Llevar mascarilla me parece bien, el alcohol me parece bien. Pero claro, ¿a mí de que me vale, si luego, en el fondo, está la fiesta y aquí no pasa nada? En diversas ocasiones, como se ha visto por la calle y gente que pasaba, que han hecho pruebas, por delante de policías y demás, y nadie les dice nada, que es un poco absurdo».

\section{Persona afiliada de entre 18-34 años.}

«En el momento en que empezamos la desescalada, yo creo que incluso fue hasta peor para las personas mayores que el propio confinamiento, porque, igual, ahí entran otra vez en juego los medios de comunicación y demás, que no daban más que noticias de cómo la gente no respetaba las distancias, de cómo la gente no llevaba las mascarillas. Y claro, nuestro colectivo, en su mayoría, no tiene resto visual funcional o el resto visual que tiene es muy poco funcional o no tiene resto visual, por lo que les entraba el agobio de "cómo voy a salir a la calle si yo no veo, si yo cuando veo a la gente ya la tengo delante y no tienen mascarilla, entonces yo no sé si hay un metro o dos" [...]. De hecho, a día de hoy, todavía hay mucha gente que prácticamente no sale de casa».

Profesional de la onCE - Trabajo social.

A esto se han de añadir las nuevas barreras que suponen las medidas introducidas para frenar el número de contagios. Estas medias son, en muchas ocasiones, visuales (pantallas informativas, marcas en el suelo o en el asfalto, separadores físicos, distanciamiento de dos metros, etc.), difíciles de apreciar por parte de una persona con discapacidad visual y que dificultan su desplazamiento por el entorno (Bertiz y Dearing, 2020). 
Cuadro 8. Comentarios extraídos de las entrevistas realizadas sobre el miedo a salir a la calle (3)

«Lo que sí que veo que se está convirtiendo en un problema es sacar las terrazas a las calles. "Es que me han puesto una terraza ahí al lado y es que no sé por dónde tengo que pasar, es que no sé si hay mucha gente o llevan las mascarillas, porque el espacio que hay es muy pequeño". Entonces la gente ha salido a la calle y, claro, hay más obstáculos físicos».

\section{Profesional de la ONCE - Trabajo social.}

Más allá del motivo por el que se tenga miedo o preocupación a salir a la calle, que, como señalan Bertiz y Dearing (2020), crea una oposición mental entre el hogar, considerado como espacio seguro, y el mundo exterior, asimilado como lo desconocido y aterrador, es importante tener en cuenta las repercusiones que esto pueda tener en las personas con discapacidad visual, ya que se está corriendo el riesgo, por un lado, de aumentar la dependencia de los demás, dejando que sean otros los que realicen las tareas que se realizaban en el exterior del domicilio, y, por otro, la pérdida de habilidades dentro del proceso de ajuste a la discapacidad, como pueden ser todas las referidas a la movilidad y orientación fuera del hogar.

Cuadro 9. Comentarios extraídos de las entrevistas realizadas sobre el miedo a salir a la calle (4)

«Eso sí, ahora voy con un poco más de miedo por la calle y a la hora de entrar a los sitios. Si puedo evitar tener que ir yo a comprar el pan y pueden ir por mí, pues mejor».

\section{Persona afiliada de entre 35 y 54 años, con movilidad reducida.}

«Sí, sí. Porque yo sola, por ejemplo, no salgo a la calle. Si he salido, ha sido o con mi padre o con mi madre. Sola no he salido».

¿Y eso lo hacías antes? ¿Antes salías sola?

«Sí, cuando iba para la residencia o si mi padre me mandaba un recado de ir al estanco, a la farmacia, eso sí lo he hecho sola».

¿Y ahora no?

«No, ahora no».

\section{Persona afiliada de entre 18-34 años.}

Otro ámbito generador de preocupaciones es el referido a la economía y al empleo. Hay que partir de que al menos el $38 \%$ de las personas con discapacidad visual declaran que la situación provocada por el coronavirus les está afectando laboral y 
económicamente mucho o bastante, porcentaje similar al de quienes están preocupados por las repercusiones que pueda tener el haber perdido el propio empleo o que lo haya perdido un familiar (Tabla 6).

Tabla 6. Preocupaciones de las personas con discapacidad visual en relación al empleo (\%)

\begin{tabular}{|l|c|}
\hline \multicolumn{1}{|c|}{ Preocupación } & Porcentaje \\
\hline $\begin{array}{l}\text { Miedo por la posibilidad de perder su empleo } \\
\text { o que algún familiar pierda el suyo }\end{array}$ & 63,4 \\
\hline $\begin{array}{l}\text { Preocupación por haber perdido su empleo } \\
\text { o que algún familiar lo haya perdido }\end{array}$ & 37,2 \\
\hline
\end{tabular}

n: 1107.

El porcentaje aumenta cuando se hace referencia a la posibilidad de futuro de perder el empleo. Al respecto, casi dos de cada tres personas encuestadas manifiestan miedo ante la posibilidad de perder el empleo actual, ya sea la propia persona o algún familiar. En muchas ocasiones, esta preocupación se manifiesta más hacia el entorno cercano que hacia uno mismo; por un lado, porque la mayoría de las personas encuestadas son personas que viven con una pensión asegurada y, por otro lado, porque parte de quienes se encuentran en la actualidad trabajando lo hacen dentro del Grupo Social ONCE, lo que, a opinión de las mismas, les aporta seguridad y tranquilidad al sentirse respaldadas por una entidad estable y que vela por los intereses y necesidades de las personas con discapacidad visual.

Cuadro 10. Comentarios extraídos de las entrevistas realizadas sobre la propia situación económica

«Eso está más crudo. Nosotros tenemos la suerte de estar en una empresa que más o menos se sostiene por todas las labores sociales que hace, y la verdad es que, para mí, eso es todo, porque por lo menos piensas "Estoy en la ONCE, es una empresa muy importante. Es muy difícil que no salga a flote. Es muy difícil". Que lo pasó mal y que yo entiendo que tendrá que recortar en cosas, y yo estoy dispuesta a recortar lo que haga falta. Me da igual.

»Pero bueno, por lo menos tienes esa cosa de decir: "Bueno, trabajo en la ONCE"».

\section{Persona afiliada de entre 18-35 años, trabajando en la ONCE.}

«Yo no soy demasiado institucionalista, la verdad, pero un diez a que ONCE nos mantuviera el sueldo íntegro, eso realmente nos dio una tranquilidad brutal».

Persona afiliada entre 36-54 años, trabajando en la ONCE. 
Teniendo en cuenta lo referido hasta el momento, no es de extrañar, por tanto, que, a la hora de preguntar sobre a qué se tiene una mayor preocupación, a los efectos de la covid-19 sobre la salud o sobre la economía y el empleo (Tabla 7), las personas con discapacidad visual se decantan más por los efectos sobre la salud, incluso en mayor medida que la población en general según el barómetro del CIS de octubre de 2020.

Tabla 7. Grado de preocupación de las personas con discapacidad visual respecto al coronavirus (\%)

\begin{tabular}{|l|r|r|}
\hline \multicolumn{1}{|c|}{ Grado de preocupación } & $\begin{array}{c}\text { Población con } \\
\text { discapacidad visual }\end{array}$ & $\begin{array}{c}\text { Conjunto de la } \\
\text { población española }\end{array}$ \\
\hline Los efectos sobre la salud & 51,9 & 40,9 \\
\hline $\begin{array}{l}\text { Los efectos sobre la economía } \\
\text { Y el empleo }\end{array}$ & 15,7 & 23,4 \\
\hline Ambos por igual & 31,0 & 34,9 \\
\hline Ni los unos ni los otros & 0,3 & 0,5 \\
\hline NS/NC & 1,1 & 0,3 \\
\hline
\end{tabular}

Fuente: encuesta ONCE ( $n$ : 1107)/Barómetro de octubre 2020, CIS ( $n$ : 2924).

Por último, respecto a las perspectivas de futuro, tal como se puede apreciar en la Tabla 8, casi dos de cada tres afiliados encuestados manifestaban que sienten intranquilidad ante la posibilidad de no recuperar la vida tal como era con anterioridad a la crisis sanitaria, ya que se considera que la misma va a tener una repercusión en la economía, tanto familiar como nacional, en la forma en la que nos relacionamos..., incluso sobrevuela el temor a futuros confinamientos como el sufrido entre los meses de marzo y mayo.

Tabla 8. Perspectivas de futuro por parte de las personas con discapacidad visual (\%)

\begin{tabular}{|l|c|}
\hline \multicolumn{1}{|c|}{ Preocupación } & \multicolumn{2}{|c|}{ Porcentaje } \\
\hline $\begin{array}{l}\text { Intranquilidad por no recuperar } \\
\text { su vida tal y como era antes }\end{array}$ & 64,7 \\
\hline
\end{tabular}

n: 1107.

Dabbagh, V.O. (2021). Cómo han vivido la pandemia las personas con discapacidad visual durante la primera y segunda ola: sus preocupaciones y su estado anímico. RED Visual: Revista Especializada en Discapacidad Visual, 77, 13-42. https://doi.org/10.53094/XBUN9999. 
Cuadro 11. Comentarios extraídos de las entrevistas realizadas sobre las perspectivas de futuro

«Yo digo a mi mujer muchas veces que es que cuando nos dejen salir, no sé si vamos a tratar a las personas igual que las tratábamos antes [...]. Sí, yo creo nos hemos vuelto muy individualistas y que nos hemos acostumbrado a relacionarnos con las personas de otra forma y hasta con otro lenguaje. Pero sé que, en parte, vamos a perder la sociabilidad, en el sentido del roce con las personas».

Persona afiliada de 55 o más años.

«Veremos ahora otra vez, veremos si no nos confinan otra vez».

Persona afiliada de entre 18-34 años.

\subsection{El estado anímico de las personas con discapacidad visual durante la pandemia}

Al hablar del estado anímico hay que tener en cuenta que este ha podido variar durante el tiempo que ha durado, y sigue durando, la pandemia. No puede considerarse que haya habido un continuo en este sentido, sino que ha habido altibajos, muchas veces influenciado por hitos dentro de la crisis: la variación de la incidencia de la covid-19, el anuncio de la prórroga del confinamiento, la aparición de una nueva ola, la esperanza de una vacuna y las dudas que se generaron sobre la misma..., lo que, tal como señalan Balluerka et al. (2020), ha producido cambios en los estados de ánimo disfóricos y eufóricos, si bien el número de personas que manifiestan estos segundos es menor.

A su vez, se debe ser consciente de que no todas las personas han vivido la situación provocada por el coronavirus de la misma manera. Hay quienes, especialmente entre los más jóvenes, lo han hecho de manera más o menos satisfactoria, en tanto que el tiempo de confinamiento les ha permitido realizar tareas que, de otra manera, no hubiesen podido, como estar más tiempo con los padres.

Otro grupo de personas, especialmente entre las personas mayores -que, por distintas circunstancias, no tienen el hábito de salir a la calle y tienen un estilo de vida sedentario-, no han notado mucho cambio en sus vidas y en sus rutinas. La existencia de personas con este perfil debe ser un aviso de posibles situaciones de soledad y aislamiento social que la crisis del coronavirus no solo las puede haber puesto en evidencia, sino que también puede haberlas agravado. 
Cuadro 12. Comentarios extraídos de las entrevistas realizadas sobre el estado anímico (1)

«Bueno, lo de la pandemia ha sido para mí..., sobre todo en el tema de cuando estábamos confinados. Eso supongo que ha sido para todos, pero sí, fue machacador, porque yo he tenido muchas temporadas como de altibajos a nivel psicológico».

\section{Persona afiliada de 55 o más años.}

«Hombre, si había una que otra bajada de contagios o de fallecimientos, pues un poco de alegría. Pero cuando ya te volvías a enterar de que volvían a subir, otra vez tristeza. Te alegras porque bajen un poco, pero, al día siguiente, otra vez han subido».

\section{Persona afiliada de entre 18-34 años.}

«Cuando empezó en febrero y marzo fue una sorpresa, no me lo esperaba. Tuve mucha confusión y mucho miedo. De verdad fue una situación muy difícil. Cuando se redujo el número de personas afectadas ya más tranquilo, y ahora en la segunda ola muy preocupado porque no nos afecte».

\section{Persona de más de 54 años con sordoceguera.}

«De hecho, durante la pandemia "Me lo estoy pasando de vicio", decía. No sufría nada».

\section{Familiar persona afiliada menor de edad.}

«Los niños lo han pasado mal; por un lado, porque no entendían nada, estaban encerrados, pero, por otro lado, han vivido una situación que jamás se hubieran planteado, y es que han estado con sus papás todos los días de la semana 24 horas, que eso también..., que si hay que sacar algo positivo es quizás eso. Hay niños que estaban disfrutando».

\section{Profesional de la ONCE - Ámbito educativo.}

«Me he encontrado todo tipo de variables, desde personas que lo vivenciaban "Bueno, pues estoy tan a gusto aquí en casa", personas mayores que normalmente su vida era un poco rutinaria y en base a actividades muy básicas, muy primarias en cuanto a que me levanto, me aseo, desayuno, pongo la tele si tengo resto visual para verla u oírla si tengo resto auditivo, y poco más. Había gente que no notaba ningún cambio significativo».

\section{Profesional de la ONCE - Ámbito sordoceguera.}

Respecto a los sentimientos negativos producidos por la situación provocada por el coronavirus, se puede apreciar (Tabla 9) cómo la mayoría de las personas con discapacidad visual ha tenido alguno durante este tiempo, especialmente preocupación o tristeza, pero también se han sentido tensas $y$, en menor medida deprimidas o enfadadas, lo que encajaría con los resultados obtenidos por el Odismet ${ }^{3}$ (Silván y Quífez, 2020) al analizar los efectos de la crisis en la población con discapacidad.

3 Observatorio sobre discapacidad y mercado de trabajo (N. del E.).

Dabbagh, V.O. (2021). Cómo han vivido la pandemia las personas con discapacidad visual durante la primera y segunda ola: sus preocupaciones y su estado anímico. RED Visual: Revista Especializada en Discapacidad Visual, 77, 13-42. https://doi.org/10.53094/XBUN9999. 
Estos sentimientos no aparecen con el mismo grado de intensidad en todos los perfiles. Así, los hombres y quienes tienen una edad comprendida entre los 18 y los 24 años son los que menos declaran tenerlos, mientras que las mujeres y quienes tienen 65 o más años son quienes más los han sentido.

Tabla 9. Sentimientos negativos de las personas con discapacidad visual durante la crisis (\%)

\begin{tabular}{|l|r|r|r|r|r|}
\hline Se ha sentido... & $\begin{array}{c}\text { Siempre o } \\
\text { casi siempre }\end{array}$ & $\begin{array}{c}\text { La mayor parte } \\
\text { del tiempo }\end{array}$ & A veces & $\begin{array}{c}\text { Nunca o } \\
\text { casi nunca }\end{array}$ & NS/NC \\
\hline Preocupada & 25,7 & 13,2 & 43,1 & 17,2 & 0,8 \\
\hline Triste & 14,4 & 9,7 & 45,9 & 28,9 & 1,1 \\
\hline Tensa & 13,0 & 8,7 & 38,9 & 38,2 & 1,2 \\
\hline Deprimida & 10,6 & 6,4 & 33,4 & 48,9 & 0,7 \\
\hline Enfadada & 8,6 & 3,5 & 38,6 & 48,2 & 1,0 \\
\hline
\end{tabular}

n: 1107.

Estos resultados son muy parecidos también a los que obtuviera el $\mathrm{CIS}^{4}$ para el conjunto de la población española al analizar los sentimientos que se han manifestado frecuentemente (es decir, que se ha señalado que se han tenido siempre o la mayor parte del tiempo), si bien, tal como se observa en la Tabla 10, las personas con discapacidad visual se muestran ligeramente más tristes y, especialmente, más deprimidas.

Tabla 10. Sentimientos negativos de las personas con discapacidad visual frecuentes durante la crisis (\%)

\begin{tabular}{|l|c|c|}
\hline $\begin{array}{c}\text { Se ha sentido siempre o la mayor } \\
\text { parte del tiempo... }\end{array}$ & $\begin{array}{c}\text { Población con } \\
\text { discapacidad visual }\end{array}$ & $\begin{array}{c}\text { Conjunto de la } \\
\text { población española }\end{array}$ \\
\hline Preocupada & 38,9 & 42,0 \\
\hline Triste & 24,1 & 21,1 \\
\hline Tensa & 21,7 & 25,6 \\
\hline Deprimida & 17,0 & 11,3 \\
\hline Enfadada & 12,1 & 14,9 \\
\hline
\end{tabular}

Fuente: encuesta ONCE ( $n$ : 1107)/Estudio sobre bienestar emocional, CIS, junio de 2020 ( $n$ : 937).

\footnotetext{
4 El CIS ha realizado distintos estudios en los que ha incluido la autovaloración del estado anímico por parte de las personas entrevistadas. Si bien el último llevado a cabo es la Encuesta sobre la salud mental de los/as españoles/as durante la pandemia de la COVID-19, de febrero de 2021, se han tomado los resultados del Estudio sobre bienestar emocional de junio de 2020 por ser el que, tanto en la redacción de las preguntas como en las opciones de respuesta, se asemejaba más al cuestionario empleado por la ONCE en su estudio.
} 
Ahondando más en estos sentimientos, se quiso analizar cómo influían otras variables en los mismos: concretamente, el vivir solo, el haber experimentado en primera persona la muerte de algún familiar, amistad o conocido, o el disponer de suficientes actividades de ocio (Tabla 11). Al respecto, se puede apreciar cómo el vivir solo tiene ligeras diferencias en algunos estados anímicos, si bien ninguna de ellas es significativa.

Por el contrario, sí que se observa cómo los sentimientos disfóricos aumentan considerablemente cuando o bien ha muerto una persona cercana al encuestado o bien no se disponen de actividades para ocupar el tiempo, aspecto muy ligado, tal y como lo confirman los discursos de las personas entrevistadas, a la percepción de estar repitiéndose siempre la misma rutina.

Tabla 11. Sentimientos negativos de las personas con discapacidad visual durante la crisis en función de vivir solo, haber experimentado la muerte de algún familiar, amistad o conocido,

y disponer de actividades de ocio (\%)

\begin{tabular}{|c|c|c|c|c|c|}
\hline $\begin{array}{c}\text { Se ha } \\
\text { sentido... }\end{array}$ & $\begin{array}{c}\text { Total } \\
\text { de la } \\
\text { población }\end{array}$ & $\begin{array}{l}\text { Personas } \\
\text { que viven } \\
\text { solas }\end{array}$ & $\begin{array}{c}\text { Personas } \\
\text { que han } \\
\text { experimentado } \\
\text { la muerte de } \\
\text { algún familiar, } \\
\text { amistad } \\
\text { o conocido }\end{array}$ & $\begin{array}{l}\text { Personas que } \\
\text { disponen de } \\
\text { suficientes } \\
\text { actividades } \\
\text { de ocio }\end{array}$ & $\begin{array}{l}\text { Personas que } \\
\text { no disponen } \\
\text { de suficientes } \\
\text { actividades } \\
\text { de ocio }\end{array}$ \\
\hline $\begin{array}{l}\text { Preocupada } \\
\text { frecuentemente }\end{array}$ & 38,9 & 37,6 & 48,6 & 35,3 & 49,3 \\
\hline $\begin{array}{l}\text { Triste } \\
\text { frecuentemente }\end{array}$ & 24,1 & 25,7 & 33,9 & 22,1 & 29,5 \\
\hline $\begin{array}{l}\text { Tensa } \\
\text { frecuentemente }\end{array}$ & 21,7 & 19,5 & 27,3 & 20,4 & 25,3 \\
\hline $\begin{array}{l}\text { Deprimida } \\
\text { frecuentemente }\end{array}$ & 17,0 & 17,1 & 23,7 & 14,0 & 25,3 \\
\hline $\begin{array}{l}\text { Enfadada } \\
\text { frecuentemente }\end{array}$ & 12,1 & 14,3 & 14,4 & 10,3 & 17,4 \\
\hline
\end{tabular}

n: 1107.

Dabbagh, V.O. (2021). Cómo han vivido la pandemia las personas con discapacidad visual durante la primera y segunda ola: sus preocupaciones y su estado anímico. RED Visual: Revista Especializada en Discapacidad Visual, 77, 13-42. https://doi.org/10.53094/XBUN9999. 
Cuadro 13. Comentarios extraídos de las entrevistas realizadas sobre el estado anímico (2)

«Hombre, muy triste cuando te vas enterando de casos que gente, a lo mejor no tan cercana, pero sí cercana y de familiares y tal, que bueno han ido alguno muriendo o enfermando y que se han ido recuperando. Y claro, si esto va a continuar... En fin».

\section{Persona afiliada de entre 36 y 54 años.}

«Varios conocidos y vecinos han fallecido y eso nos ha afectado mucho. Eso nos obliga a estar en casa».

\section{Persona afiliada de más de 54 años con sordoceguera.}

«La verdad es que ha habido días que me he metido en la cama y no he salido de la cama, porque era una rutina de todos los días lo mismo. Te levantas y todos los días lo mismo».

\section{Persona afiliada de entre 36 y 54 años.}

«Esto ya cansa, algunas veces esto ya es demasiado».

\section{Persona afiliada de entre 18-34 años.}

Así mismo, tal y como adelantaba la European Union Agency for Fundamental Rights (2020), una parte de las personas con discapacidad visual, el 16,6\%, han visto cómo servicios que son considerados de vital importancia han sido suspendidos o interrumpidos, lo que ha afectado considerablemente a su estado de ánimo (Tabla 12); de hecho, es uno de los factores que más influye en el surgimiento de sentimientos negativos.

Tabla 12. Sentimientos negativos de las personas con discapacidad visual durante la crisis en función de haber recibido alguno de los servicios de la ONCE (\%)

\begin{tabular}{|l|r|r|}
\hline \multicolumn{1}{|c|}{ Se ha sentido... } & $\begin{array}{r}\text { No ha dejado de recibir } \\
\text { algún servicio vital }\end{array}$ & $\begin{array}{c}\text { Ha dejado de recibir } \\
\text { algún servicio vital }\end{array}$ \\
\hline Preocupada & 80,2 & 91,8 \\
\hline $\begin{array}{l}\text { Preocupada } \\
\text { frecuentemente }\end{array}$ & 35,4 & 57,6 \\
\hline Triste & 67,8 & 81,5 \\
\hline Triste frecuentemente & 21,5 & 37,5 \\
\hline
\end{tabular}

Dabbagh, V.O. (2021). Cómo han vivido la pandemia las personas con discapacidad visual durante la primera y segunda ola: sus preocupaciones y su estado anímico. RED Visual: Revista Especializada en Discapacidad Visual, 77, 13-42. https://doi.org/10.53094/XBUN9999. 


\begin{tabular}{|l|r|r|}
\hline \multicolumn{1}{|c|}{ Se ha sentido... } & $\begin{array}{c}\text { No ha dejado de recibir } \\
\text { algún servicio vital }\end{array}$ & $\begin{array}{c}\text { Ha dejado de recibir } \\
\text { algún servicio vital }\end{array}$ \\
\hline Tensa & 58,2 & 73,4 \\
\hline Tensa frecuentemente & 19,4 & 33,2 \\
\hline Deprimida & 46,8 & 70,1 \\
\hline Deprimida frecuentemente & 14,8 & 28,3 \\
\hline Enfadada & 47,8 & 66,3 \\
\hline Enfadada frecuentemente & 9,6 & 24,5 \\
\hline
\end{tabular}

$n: 1107$.

Paralelamente a estos factores, y teniendo en cuenta que la actividad de la ONCE hacia sus afiliados siguió funcionando, tanto durante el confinamiento como después de este -eso sí, modificando en lo necesario la forma de llevarlos a cabo-, se quiso comprobar si esta actividad, valorada como muy buena o buena por el $76,5 \%$ de las personas encuestadas, había tenido alguna repercusión en el estado anímico de los afiliados. Para ello, primeramente, de la amplia gama de servicios prestados por la ONCE, se les preguntó si habían recibido en concreto cuatro de ellos (contacto telefónico, apoyo psicológico, servicio de voluntariado o acceso al ClubONCE). ${ }^{5}$ Casi el $80 \%$ declaró haber recibido alguno de estos servicios, siendo el más frecuente el contacto telefónico (75,8\%).

Con este dato, y al cruzarlo con los estados anímicos anteriormente analizados, se pudo comprobar que si bien había una ligera diferencia entre quienes habían recibido algún servicio y quienes no, esta era muy reducida, salvo en el caso de quienes señalaban sentirse deprimidos, en cuyo caso, quienes no habían recibido alguno de los servicios mencionados manifestaban en mayor proporción el haber sentido depresión -más de 10 puntos porcentuales de diferencia-, siendo esta estadísticamente significativa (Tabla 13). Lo que viene a confirmar la idea manifestada por Javed et al. (2020) cuando afirmaban que una simple llamada telefónica durante el confinamiento puede suponer una ayuda para la salud mental de las personas y proporcionarles consuelo.

5 ClubONCE es el espacio web enfocado en aportar información, servicios y recursos específicos para personas afiliadas a la ONCE (Biblioteca Digital, películas audiodescritas, tutoriales, noticias de interés, etc.). Se puede acceder desde la web de la ONCE o directamente en club.once.es.

Dabbagh, V.O. (2021). Cómo han vivido la pandemia las personas con discapacidad visual durante la primera y segunda ola: sus preocupaciones y su estado anímico. RED Visual: Revista Especializada en Discapacidad Visual, 77, 13-42. https://doi.org/10.53094/XBUN9999. 
Tabla 13. Sentimientos negativos de las personas con discapacidad visual durante la crisis en función de haber recibido alguno de los servicios de la ONCE (\%)

\begin{tabular}{|l|r|r|}
\hline \multicolumn{1}{|c|}{ Se ha sentido... } & $\begin{array}{c}\text { Personas } \\
\text { usuarias }\end{array}$ & $\begin{array}{c}\text { Personas no } \\
\text { usuarias }\end{array}$ \\
\hline Preocupada frecuentemente & 37,9 & 42,9 \\
\hline Triste frecuentemente & 23,6 & 25,7 \\
\hline Tensa frecuentemente & 21,6 & 22,1 \\
\hline Deprimida frecuentemente & 14,9 & 25,2 \\
\hline Enfadada frecuentemente & 11,9 & 12,8 \\
\hline
\end{tabular}

n: 1107.

Junto a los estados anímicos analizados hasta el momento, se consideró relevante estudiar la soledad de las personas afiliadas durante el confinamiento y después de este.

Frecuentemente, se utiliza para una primera toma de contacto con este fenómeno el porcentaje de hogares unipersonales, siendo conscientes de que vivir solos no significa necesariamente tener sentimientos de soledad. Al respecto, y en relación a la muestra del estudio, prácticamente una de cada cinco personas encuestadas declaró que vivían solas. Por el contrario, las personas que declaran haber sentido soledad en algún momento (Tabla 14), independientemente de su forma de convivencia, ascienden hasta el 33,9\%, de los cuales para el $42 \%$ este sentimiento ha estado presente siempre o la mayor parte del tiempo.

Esta percepción subjetiva de la soledad se ha visto influenciada por la edad y el sexo, de tal manera que los afiliados de entre 18 y 34 años y los hombres son los que en menor medida han manifestado haberse sentido solos, mientras que, por el contrario, quienes declaraban en mayor proporción sentir dicha soledad eran los afiliados de 75 o más años y las mujeres.

Así mismo, comparando los resultados obtenidos con los datos de la población española en su conjunto, se puede apreciar cómo además de ser ligeramente más frecuente la soledad en la población con discapacidad visual, esta es más intensa. En ello, entre otros factores, influye la accesibilidad, entre otras, de las nuevas tecnologías, tan importantes durante el confinamiento, ya que la falta de la misma ha 
supuesto una barrera adicional en las personas con discapacidad visual a la hora de comunicarse con los demás, a la hora de acceder a distintos servicios (Zayed et al., 2020) o incluso a la hora de realizar distintas gestiones; pero también otros aspectos, como el no contar con los apoyos habituales (Bertiz y Dearing, 2020), como es, en el caso de las personas con sordoceguera, las y los mediadores y voluntarios.

Tabla 14. Sentimiento de soledad en la población durante la crisis (\%)

\begin{tabular}{|l|r|r|r|r|r|}
\hline \multicolumn{1}{|c|}{ Perfil } & $\begin{array}{c}\text { Siempre } \\
\text { o casi } \\
\text { siempre }\end{array}$ & $\begin{array}{c}\text { La mayor } \\
\text { parte del } \\
\text { tiempo }\end{array}$ & A veces & $\begin{array}{c}\text { Nunca o } \\
\text { casi nunca }\end{array}$ & NS/NC \\
\hline $\begin{array}{l}\text { Población con } \\
\text { discapacidad visual }\end{array}$ & 8,9 & 5,2 & 19,8 & 65,1 & 0,9 \\
\hline $\begin{array}{l}\text { Hombres con } \\
\text { discapacidad visual }\end{array}$ & 6,0 & 3,6 & 18,3 & 71,5 & 0,6 \\
\hline $\begin{array}{l}\text { Mujeres con } \\
\text { discapacidad visual }\end{array}$ & 11,6 & 6,7 & 21,1 & 59,3 & 1,2 \\
\hline $\begin{array}{l}18-34 \text { años con } \\
\text { discapacidad visual }\end{array}$ & 3,5 & 1,2 & 19,8 & 74,4 & 1,2 \\
\hline $\begin{array}{l}75 \text { o más años con } \\
\text { discapacidad visual }\end{array}$ & 13,1 & 5,2 & 15,7 & 65,0 & 0,9 \\
\hline Población española & 4,3 & 7,4 & 18,5 & 69,4 & 0,9 \\
\hline
\end{tabular}

Fuente: encuesta ONCE ( $n$ : 1107)/Estudio sobre bienestar emocional, CIS, junio de 2020 ( $n$ : 937).

Al igual que se hiciera con los anteriores estados de ánimo analizados (preocupación, tristeza, depresión, enfado, estar tenso), se analizaron distintos factores para comprobar cómo influenciaban el sentimiento de soledad (Tabla 15). En primer lugar, se analizaron los efectos de vivir solo en relación a la soledad. En este sentido, se puede apreciar cómo la soledad aumenta considerablemente en quienes viven sin compañía, ya que más de la mitad afirma haber sentido soledad en algún momento y casi el $30 \%$ la ha sentido frecuentemente.

A pesar de existir una fuerte relación entre el sentimiento de soledad y el vivir o no solo, no es una correspondencia perfecta, de tal manera que hay personas que, aun viviendo solas, no han manifestado sentir soledad, $y$, al contrario, personas que han convivido con otra gente durante los meses de la crisis y que, a pesar de ello,

Dabbagh, V.O. (2021). Cómo han vivido la pandemia las personas con discapacidad visual durante la primera y segunda ola: sus preocupaciones y su estado anímico. RED Visual: Revista Especializada en Discapacidad Visual, 77, 13-42. https://doi.org/10.53094/XBUN9999. 
se han sentido solas, lo que demuestra que dentro de la soledad hay varios factores que entran en juego, que no se puede hablar de una única soledad y que, incluso, se puede hacer alusión a soledades compartidas cuando las mismas se producen en el mismo núcleo residencial.

Cuadro 14. Comentarios extraídos de las entrevistas realizadas sobre la soledad

«Creo que lo que más notas es que, aunque estés acompañada y sabes que están ahí, creo que noto soledad».

\section{Persona afiliada de entre 18-35 años, con movilidad reducida.}

«Vivo con mi mujer e hijo, pero tengo sentimiento de soledad».

\section{Persona afiliada de entre 35 y 54 años con sordoceguera.}

«Hay gente que vivía sola y no se sentía sola, y hay gente que vivía acompañada y se sentía muy sola. Al final, no es una cuestión de colectivos, es una cuestión de actitud personal. Entonces, hemos tenido de todo. Gente que vivía sola y se sentía superacompañada porque se pasaba el día hablando por teléfono. A veces, ni siquiera tenían un smartphone que tuviera WhatsApp o tal [...]. Y había gente que se sentía muy sola, que todo el día lo mismo, todo el día la radio, que es que no se puede hacer nada, y empezabas a escarbar un poquito y veías que recibían llamadas, que estaban pendientes de ellos, pero se sentían solas igualmente. Y había gente que prácticamente no hablaba con nadie y no se sentía sola porque era su forma de vida, porque era así antes».

Profesional de la onCE - Trabajo social.

Tabla 15. Sentimiento de soledad en las personas con discapacidad visual durante la crisis en función de distintas variables (\%)

\begin{tabular}{|l|r|r|}
\hline \multicolumn{1}{|c|}{ Variables } & Sentirse solo & $\begin{array}{c}\text { Sentirse solo } \\
\text { frecuentemente }\end{array}$ \\
\hline General & 33,9 & 14,1 \\
\hline Vive sola & 54,3 & 29,1 \\
\hline Vive acompañada & 31,4 & 10,2 \\
\hline Ha muerto algún familiar, amistad o conocido & 38,7 & 17,4 \\
\hline No ha muerto algún familiar, amistad o conocido & 31,9 & 12,7 \\
\hline Tener suficientes actividades de ocio & 30,9 & 12,8 \\
\hline No tener suficientes actividades de ocio & 42,7 & 18,1 \\
\hline
\end{tabular}

Dabbagh, V.O. (2021). Cómo han vivido la pandemia las personas con discapacidad visual durante la primera y segunda ola: sus preocupaciones y su estado anímico. RED Visual: Revista Especializada en Discapacidad Visual, 77, 13-42. https://doi.org/10.53094/XBUN9999. 


\begin{tabular}{|l|r|r|}
\hline \multicolumn{1}{|c|}{ Variables } & Sentirse solo & \multicolumn{1}{|c|}{$\begin{array}{c}\text { Sentirse solo } \\
\text { frecuentemente }\end{array}$} \\
\hline Uso diario de Internet & 32,3 & 10,8 \\
\hline NO uso diario de Internet & 35,5 & 17,4 \\
\hline $\begin{array}{l}\text { NO ha dejado de recibir servicios considerados } \\
\text { Como vitales }\end{array}$ & 32,1 & 12,6 \\
\hline $\begin{array}{l}\text { Ha dejado de recibir servicios considerados } \\
\text { como vitales }\end{array}$ & 44,0 & 22,8 \\
\hline Personas usuarias de servicios de la ONCE & 33,1 & 13,2 \\
\hline Personas No usuarias de servicios de la ONCE & 37,2 & 18,1 \\
\hline
\end{tabular}

n: 1107.

Junto al hecho de vivir solo, que es la variable que más incide en el sentimiento de soledad, también se ha visto cómo otros factores influyen en el mismo. El contar con suficientes actividades de ocio, hacer uso de Internet a diario o recibir determinados servicios de atención hacen que este sentimiento sea menos frecuente y que se manifieste en menor grado de intensidad, mientras que el vivir en primera persona la muerte de algún familiar, amistad o conocido y, sobre todo, el dejar de recibir servicios que se consideran vitales, provocan exactamente lo contrario.

\section{Conclusiones}

En este artículo se han presentado los principales resultados sobre las preocupaciones y el estado de ánimo de las personas con discapacidad visual dentro de un estudio más amplio que la ONCE realizó a finales de 2020 entre sus afiliados.

Se ha puesto en evidencia cómo existe un grado de preocupación alto respecto al coronavirus entre las personas con ceguera o con deficiencia visual, una preocupación que no solo tiene que ver con la propia salud personal, sino que hay varios factores que entran en juego, como la salud de las personas del entorno cercano, la preocupación por el acceso a la atención sanitaria, la pérdida de algún empleo dentro de la unidad familiar, la situación económica, etc.

Importante resaltar la preocupación que se ha manifestado por salir a la calle, que, si bien es una preocupación compartida con el conjunto de la población, especialmente

Dabbagh, V.O. (2021). Cómo han vivido la pandemia las personas con discapacidad visual durante la primera y segunda ola: sus preocupaciones y su estado anímico. RED Visual: Revista Especializada en Discapacidad Visual, 77, 13-42. https://doi.org/10.53094/XBUN9999. 
con la población más vulnerable a la covid-19, en el caso de las personas con ceguera o con deficiencia visual esta tiene dos particularidades. En primer lugar, porque a estas se les añaden una serie de elementos que aumentan la dificultad para un normal desplazamiento y en condiciones de seguridad, como son la dificultad de mantener el distanciamiento social, la imposibilidad de percibir los elementos visuales destinados para mantener dicho distanciamiento o elementos inusuales en los recorridos aprendidos, la necesidad de tocar para relacionarse con el entorno y extraer información de este, etc.

Por otro lado, la preocupación por salir a la calle puede llevar aparejada una creciente inactivad fuera del hogar, y con ella una mayor dependencia hacia otras personas y una pérdida de las habilidades adquiridas en cuanto a movilidad y desplazamientos se refieren.

También se ha señalado cómo el estado anímico de las personas con discapacidad visual ha podido sufrir altibajos, pasando de estados eufóricos, los menos usuales, a estados disfóricos, siendo los más habituales la preocupación, la tristeza o el estar tenso y, en un segundo nivel, la soledad, la depresión o el enfado. Sobre estos estados se ha visto cómo hay determinados factores que los agravan o atenúan, como es el hecho de recibir servicios de apoyo, el contar con suficientes actividades de ocio, el haber vivido en primera persona la muerte de algún familiar, amistad o conocido, y, además, en el caso concreto de la soledad, el vivir solo o la frecuencia de uso de Internet.

En todos los estados analizados, un factor determinante ha sido el dejar de recibir un servicio que se considerase necesario o vital, lo que refuerza la advertencia de la European Union Agency for Fundamental Rights (2020) sobre el riesgo de la suspensión o interrupción de los servicios y apoyos para las personas con discapacidad, ya que no solamente afectan a su salud física, sino también a su salud emocional.

Por otro lado, en cuanto a estado anímico se refiere, es necesario poner el foco de atención en aquellas personas que han declarado que su estilo de vida no ha sufrido variaciones con el inicio de la pandemia, ya que tienen poca costumbre de salir a la calle o de relacionarse con gente fuera del hogar, puesto que podrían estar manifestando situaciones de aislamiento y soledad que necesitarían un análisis en mayor profundidad. 
Por último, también es importante recalcar cómo algunos de los estados de ánimo analizados, concretamente los sentimientos de tristeza, depresión y soledad, son más frecuentes y más intensos en las personas con discapacidad visual que en la población en conjunto, siendo una de las posibles causas de esta diferencia el mayor aislamiento social. Como señalan Zayed et al. (2020), si bien la población en general ha podido experimentar niveles altos de estrés u otros sentimientos negativos, hay que tener presente el plus añadido que supone para las personas con discapacidad visual la exclusión social, la inaccesibilidad a los servicios y la falta de apoyos.

\section{Referencias bibliográficas}

Ammar, A., Trabelsi, K., Brach, M., Chtourou, H., Boukhris, O., Masmoudi, L., Bouaziz, B., Bentlage, E., How, D., Ahmed, M., Mueller, P., Mueller, A., Hammouda, O., Paineiras-Domingos, L.L., Braakman-Jansen, L., Wrede, C., Bastoni, S., Soares, C., Mataruna, L., ... Hoekelmann, A. (2021). Effects of home confinement on mental health and lifestyle behaviours during the COVID-19 outbreak: Insight from the «ECLBCOVID19» multi countries survey [PDF]. Biology of Sport, 38(1), 9-21. https://doi. org/10.5114/biolsport.2020.96857.

Balluerka, N., Gómez, J., Hidalgo, M. D., Gorostiaga, A., Espada, J.P., Padilla, J. L., y Santed, M.Á. (2020). Las consecuencias psicológicas de la CoVID-19 y el confinamiento [PDF]. Servicio de Publicaciones de la Universidad del País Vasco.

Bertiz, F., y Dearing, T. (2020). Voice of the customer: living with sight loss through lockdown in the United Kingdom (UK) [Word]. Royal National Institute of Blind People.

Brennan, C.S. (2020). Disability rights during the pandemic: a global report on findings of the COVID-19 Disability Rights Monitor [PDF]. COVID-19 Disability Rights Monitor.

Cénat, J.M., Blais-Rochette, C., Kokou-Kpolou, C.K., Noorishad, P.G., Mukunzi, J.N., McIntee, S.E., Dalexis, R.D., Goulet, M.A., y Labelle, P.R. (2021). Prevalence of symptoms of depression, anxiety, insomnia, posttraumatic stress disorder, and psychological distress among populations affected by the COVID-19 pandemic: a systematic review and meta-analysis [PDF]. Psychiatry Research, 295, 113599, 16 pp. https://doi.org/10.1016/j.psychres.2020.113599.

Dabbagh, V.O. (2021). Cómo han vivido la pandemia las personas con discapacidad visual durante la primera y segunda ola: sus preocupaciones y su estado anímico. RED Visual: Revista Especializada en Discapacidad Visual, 77, 13-42. https://doi.org/10.53094/XBUN9999. 
Centro de Investigaciones Sociológicas (2020a). Barómetro de octubre 2020: avance de resultados [PDF]. Estudio n.o 3296, octubre de 2020. Centro de Investigaciones Sociológicas.

Centro de Investigaciones Sociológicas (2020b). Estudio sobre bienestar emocional (piloto CATI) [PDF]. Estudio n. ${ }^{0}$ 3285, junio de 2020. Centro de Investigaciones Sociológicas.

Centro de Investigaciones Sociológicas (2021). Encuesta sobre la salud mental de los/as españoles/as durante la pandemia de la COVID-19: avance de resultados [PDF]. Estudio n. ${ }^{0}$ 3312, febrero de 2021. Centro de Investigaciones Sociológicas.

European Union Agency for Fundamental Rights (2020). Coronavirus pandemic in the EU: fundamental rights implications [PDF]. Boletín n. 1. Publications Office of the European Union. https://doi.org/10.2811/009602.

Gordon, K.D. (2020). COVID-19 Survey Report: the impact of the COVID-19 pandemic on canadians who are blind, deaf-blind, and partially-sighted [PDF], 30 de abril de 2020. Canadian Council of the Blind National Office.

Jacobson, W.H. (1993). Art and science of teaching orientation and mobility to persons with visual impairments. AFB Press.

Javed, B., Sarwer, A., Soto, E. B., y Mashwani, Z. (2020). The coronavirus (COVID-19) pandemic's impact on menthal health [PDF]. The International Journal of Health Planning and Management, 35(5), 993-996. https://doi.org/10.1002/hpm.3008.

Marroquín, B., Vine, V., y Morgan, R. (2020). Mental health during the COVID-19 pandemic: effects of stay-at-home policies, social distancing behavior, and social resources [PDF]. Psychiatry Research, 293, 113419, 9 pp. https://doi.org/10.1016/j. psychres.2020.113419.

Organización Mundial de la Salud (2020). Consideraciones relativas a la discapacidad durante el brote de COVID-19 [PDF]. Organización Mundial de la Salud, Oficina Regional para las Américas.

Ortiz, P. (coord., ed.) (2011). Discapacidad visual y autonomía personal: enfoque práctico de la rehabilitación [PDF]. Organización Nacional de Ciegos Españoles.

Dabbagh, V.O. (2021). Cómo han vivido la pandemia las personas con discapacidad visual durante la primera y segunda ola: sus preocupaciones y su estado anímico. RED Visual: Revista Especializada en Discapacidad Visual, 77, 13-42. https://doi.org/10.53094/XBUN9999. 
Rosenblum, L.P., Chanes-Mora, P., McBride, C.R., Flewellen, J., Nagarajan, N., Nave, R., y Swenor, B. (2020). Flatten inaccessibility: impact of COVID-19 on adults who are blind or have low vision in the United States [PDF]. American Foundation for the Blind.

Silván, C., y Quífez, L. E. (2020). Efectos y consecuencias de la crisis de la COVID-19 entre las personas con discapacidad [PDF]. Observatorio sobre discapacidad y mercado de trabajo.

Zayed, Y., Galarza, R., Acuña, M.P., y Dard, B. (2020). Amplifying voices: our lives, our say; Learning from COVID-19 through the experiences of blind and partially sighted persons across the world [PDF]. World Blind Union.

Víctor Omar Dabbagh Rollán. Sociólogo. Asesoría de Servicios Sociales de la ONCE, Dirección General de la ONCE. Carrera de San Jerónimo, 28; 28014 Madrid (España). Correo electrónico: vdr@once.es. 